Courrier du Centre international Blaise Pascal

$27 \mid 2005$

Varia

\title{
Pascal en Italie : une décennie d'études
}

\section{Maria Vita Romeo}

\section{OpenEdition}

Journals

Édition électronique

URL : http://journals.openedition.org/ccibp/514

DOI : $10.4000 /$ ccibp. 514

ISSN : 2493-7460

\section{Éditeur}

Centre international Blaise Pascal

\section{Édition imprimée}

Date de publication : 5 décembre 2005

Pagination : $39-50$

ISBN : 2-84516-304-5

ISSN : 0249-6674

Référence électronique

Maria Vita Romeo, «Pascal en Italie : une décennie d'études », Courrier du Centre international Blaise Pascal [En ligne], 27 | 2005, mis en ligne le 02 décembre 2015, consulté le 20 avril 2019. URL : http:// journals.openedition.org/ccibp/514 ; DOI : 10.4000/ccibp.514

Ce document a été généré automatiquement le 20 avril 2019

Centre international Blaise Pascal 


\title{
Pascal en Italie : une décennie d'études
}

\author{
Maria Vita Romeo
}

1 Depuis quelques années, on assiste en Italie à une renaissance des études pascaliennes. Sur ce point, il suffit de mentionner les récents congrès internationaux qui, en trois années consécutives, ont été organisés dans les universités italiennes : le premier en 2002 à Rome, organisé par Benedetta Papasogli à la Libera Università Maria SS Assunta ", et les deux autres, en 2003 et 2004, par Giuseppe Pezzino, à l'Université de Catane. Trois initiatives considérables, dans la mesure où elles témoignent de toute une floraison d'intérêt à l'égard de Pascal en Italie, ainsi que de la vitalité et de la fécondité des rapports actuels entre les chercheurs français et italiens. D'autre part, de telles initiatives trouvent leur raison d'être et leur fondement sur un riche terrain d'études et de publications qui, depuis les dix dernières années, ont en Italie « relu » les divers aspects de la pensée pascalienne.

2 C'est précisément à ces récentes études parues en Italie que je ferai référence pour tenter de comprendre si, dans une certaine mesure, cette renaissance incontestable pourrait être liée à un besoin culturel nouveau de "révision critique », ou, pour mieux dire, à la nécessité de se rapprocher du penseur clermontois avec une nouvelle disposition culturelle qui, d'un côté, cherche à dépasser aussi bien l'interprétation "tragique » de Goldmann ${ }^{1}$ que celle, "anti-humaniste », d'Augusto Del Noce ${ }^{2}$, et de l'autre, repousser cette espèce de «vulgate » réductionniste qui, pendant de nombreuses années, a écrasé Pascal, exclusivement sur le plan du scepticisme, de l'irrationalisme et du mysticisme religieux.

3 Ce n'est pas par hasard qu'ont récemment vu le jour en Italie quelques études qui privilégient le Pascal scientifique, dans deux perspectives: d'une part, découvrir l'existence de valeurs philosophiques dans les écrits scientifiques, en vue de surpasser le vieux dualisme récurrent entre un Pascal scientifique et un Pascal philosophe irrationaliste, entre un Pascal mondain et un Pascal chrétien janséniste ; et d'autre part, individualiser, dans le clavier unitaire d'ensemble de l'activité pascalienne, l'actualité du message d'un grand moderne qui, tout en reconnaissant la valeur et le rôle de la raison et 
de la science, revendique la valeur d'un homme, qui n'est pas seulement raison calculante, et d'un Dieu, qui n'est pas seulement le « Dieu des philosophes».

C'est pourquoi je traiterai pour premier point de mon compte-rendu le courant critique qui affirme l'unité du développement de la pensée pascalienne, et met en relief les éléments de continuité qui existent entre le Pascal savant et le Pascal chrétien, mettant par conséquent en crise la thèse qui taxe d'irrationalisme la philosophie pascalienne. Je ferai ensuite référence aux études qui traitent de Pascal penseur politique et qui lancent l'idée d'un Pascal «critique de la raison politique », pour parvenir à la définition d'un Pascal qui «caractérise les perspectives d'une politique possible et bonne». Enfin, j'achèverai mon enquête en passant en revue les études qui mettent en lumière la valeur de l'éthique pascalienne, une éthique du cœur, dont on ne peut faire abstraction dès lors que l'on analyse un aspect, qu'il soit philosophique ou scientifique, de la pensée pascalienne.

\section{L'unité de Pascal penseur}

5 La thèse du développement unitaire de la pensée de Pascal est le dénominateur commun de certaines études qui ont récemment rejeté le vieux cliché d'un Pascal qui, dans l'élan enthousiaste de sa conversion, abandonne avec mépris les études scientifiques et les intérêts mondains pour se réfugier définitivement et exclusivement dans les méditations et dans les pratiques de la religion et de la foi. C'est dans l'optique de cette thèse unitaire que se situent les deux importants volumes d'Alberto Peratoner ${ }^{3}$, qui examine la genèse de ce cliché dualiste entre Pascal savant et le Pascal penseur chrétien, cliché qui a germé dans le cercle même de famille et des amis de Blaise et qui perdure jusqu'au XIX ${ }^{\mathrm{e}}$ siècle. Si bien que le fait que Leibniz lui-même puisse déplorer l'influence négative exercée par les " préjugés de Rome » sur la pensée pascalienne, ne peut étonner. En résultera d'autre part un jugement également partial des romantiques qui, dans le désir fougueux de réévaluer Pascal religieux, négligent de manière tout aussi unilatérale Pascal savant, ouvrant les portes à la lecture irrationaliste et sceptique des thèses pascaliennes.

Il faut en outre reconnaître à Peratoner le mérite d'avoir tenté « dépasser » la dimension du caractère fragmentaire des Pensées par le moyen d'une reconstruction de l'horizon unitaire du projet de l'œuvre de Pascal, lequel recourt tout de même à une raison qui « se fait architectonique dans la détermination les lignes portantes de l'argumentation, de leurs points forts, de l'articulation conceptuelle du discours, (...) jusqu'à la fermeté logique de tous les passages posés en être par la « raison raisonnante ${ }^{4} »$. Une vision qui ne concorde certes pas avec celles qui restent encore liées à l'image d'un Pascal « tourmenté » ou à celle d'un Pascal divisé entre science et la foi.

7 C'est sur la nécessité de reconstruire l'authenticité de la pensée pascalienne que Benedetta Papasogli attire l'attention, dans son Introduction à la nouvelle édition italienne des Pensées ${ }^{6}$. Cette spécialiste italienne met en évidence la nécessité de reconstruire le plan de l'Apologie, un plan existant déjà, dont la coordination est exactement reconstituée par Philippe Sellier, qui met en évidence le «mouvement continu» de l'œuvre pascalienne, où « la raison est impliquée jusqu'au bout ». Ainsi, à ce que les philosophes des Lumières affirmaient comme une "pensée faible ", se substitue une "pensée forte ", dont l'Apologie semble se constituer et se nourrir; il est possible en effet d'y trouver, même si c'est à un autre niveau, la rigueur de la science, de l'esprit de géométrie, et la souplesse de l'esprit de finesse, tous deux mis au service de la «recherche des vérités 
ultimes sur la question de Dieu, qui est aussi, de manière indissociable, la question de l'homme ${ }^{7}$ ».

8 Très significative est la contribution de Paolo Francesco Adorno qui, dans son essai $L a$ ragione ordinata $a^{8}$, partant d'un rapprochement entre Augustin et Pascal, relève chez ce dernier « le bon sens d'une raison ordonnée », c'est-à-dire capable, en dépit de ses limites, d'imposer un ordre, de le définir et de le généraliser. Au terme d'une analyse plus attentive de la méthodologie et des œuvres de Pascal, il apparaît de façon évidente que la raison et ses procédés ne restent pas totalement exclus du domaine de la religion. En effet, s'il est vrai que la raison se révèle incapable de démontrer la vérité des principes premiers, il est aussi vrai, cependant, que cette même « incapacité » de la raison nous fait prendre conscience de notre instabilité et de l'insuffisance de nos connaissances. Et cette prise de conscience est donc un point de départ qui doit et qui peut être dépassé grâce à l'acquisition de points de vue divers, à coup sûr pas basés sur le sens commun'.

9 Dans cette perspective s'insère Lucia Pezza, qui s'engage dans une réflexion sur les «tentations du fini " (la concupiscence, l'autosuffisance, le pouvoir), tentations entendues comme limites, comme horizons définis, à partir desquels cependant nous sommes poussés à nous repenser nous-mêmes : «S'il est vrai que toutes nos voies sont voies de finitude, il est également vrai que « les portiques de la Jérusalem sainte », bien que séparées par un abîme, ne sont pas hors d'atteinte, parce que ce qui nous sépare de ceux-ci est la mesure de notre portée ${ }^{10} »$. Et, dans l'accomplissement de cette portée, on ne peut que reconnaître le rôle sans conteste important, même s'il n'est pas primordial, de la raison, qui, en partant justement de la tentation de franchir toute frontière, finit par reconnaître ses propres limites et, par conséquent, par admettre l'existence de choses qu'elle ignore, donc qui la surpassent.

10 Vaincre les «tentations du fini » ne signifie pas toutefois en perdre le souvenir, mais plutôt apprendre à regarder avec des yeux différents, ayant bien en tête cette humilité née précisément de la limite - grâce à laquelle il est possible de se sauver. De sorte que Pascal recourt certainement à la raison, afin de surmonter les tentations du fini ; mais, pour regagner la « limite » entendue comme conscience d'humilité, il se sert des « raisons du cœur ${ }^{11} »$.

11 La nature restera donc incompréhensible à l'homme, tant celui-ci ne saura dépasser la connaissance fondée sur le sens commun. D'où la nécessité de repousser les limites de l'anthropologie et de l'ontologie à une épistémologie, en recherchant à cette fin un point de vue supérieur qui aidera l'homme à mieux comprendre ou sa propre nature, ou la nature même. C'est en cela que consiste la dialectique pascalienne, en cette nécessité de conquérir un "point de vue supérieur " grâce auquel il est possible d'ordonner la connaissance ${ }^{12}$. Cette nécessité de conquérir un point de vue supérieur, qui se trouve à la base de toutes les recherches scientifiques de Pascal, ne concerne pourtant pas seulement la connaissance scientifique: en effet, elle engage aussi le domaine moral. Ici, bien évidemment, la primauté revient au cœur, unique organe qui nous permet de saisir ce point fixe par excellence sur lequel régler tout notre comportement et qui, comme on le déduit de l'Entretien, se trouve dans les Évangiles.

Dans cette ligne se situent aussi mes deux derniers essais ${ }^{13}$, qui soulignent la nécessité de reconsidérer et de réévaluer philosophiquement les travaux scientifiques de Pascal, pour parvenir ainsi à une vision d'ensemble plus complète et exhaustive de la palette pascalienne toute entière. Vision incontestablement éloignée de la conception dualiste, bien dépassée, d'un Pascal dimidiatus, conception qui a sans doute indirectement poussé, 
dans le passé, la majorité des chercheurs italiens à négliger les travaux scientifiques du penseur clermontois. Et si hier, en Italie du moins, les "chefs-d'œuvre pascaliens " étaient par-dessus tout les Pensées ou les Provinciales, aujourd'hui, à côté d'eux, se rangent à juste titre les Traités sur la Roulette, un chef-d'œuvre peut-être un peu trop négligé dans le passé. À mon sens, en effet, ces Traités ne représentent pas seulement l'expression la plus complète et la plus mûre de la pensée mathématique de Pascal, mais rappellent directement nécessairement, pour leur pleine compréhension, deux points cardinaux de son itinéraire philosophico-religieux : la théorie des trois ordres, et le concept de cœur. Deux points cardinaux, je le répète, qui restent à la base de la recherche pascalienne entière, et qui en témoignent la substantielle et indéniable unité de développement.

Ainsi apparait convaincante l'affirmation de Giuseppe Brescia qui, ayant traduit les travaux mathématiques de Pascal en italien ${ }^{14}$, n'a pu éviter de «mettre en lumière l'unité de la pensée pascalienne ${ }^{15}$ ", afin de retrouver ce dénominateur commun qui relie harmonieusement les instances éthiques aux épistémologiques ${ }^{16}$.

Enfin dans son récent Pascal e i suoi problemi ${ }^{17}$, Bernardo Razzotti oppose « son » Pascal au Pascal romantique de Chateaubriand, au sceptique de Cousin, au Pascal ni philosophe rationaliste ni sceptique de Brunschvicg, et au Pascal «sublime misanthrope» de Voltaire. En d'autres termes, Razzotti se réfère à un Pascal plus proche de l'homme moderne, qui, malgré un certain "manque de cohérence », progresse toujours suivant une ligne de continuité et de développement unitaire. Par conséquent, « il est impossible de dissocier Pascal philosophe de Pascal spécialiste de géométrie et de mathématiques, de Pascal critique de la pensée éthique, religieuse et politique ${ }^{18} »$.

On ne s'étonnera pas si, derrière cette «nouvelle tendance » à affirmer un Pascal «en relief » et "à la vie multiforme », c'est-à-dire un Pascal qui «va aux choses », Domenico Bosco nous propose la traduction italienne de l'œuvre de Ravaisson, La filosofia di Pascal, en l'étoffant d'un gros essai introductif, qui nous aide à mieux comprendre «l'autre Pascal», dont Ravaisson se fait le porte-parole, manifestant au fond l'exigence de redécouvrir un Pascal plus proche de nous, « un Pascal que chacun aurait voulu écrire ».

Ainsi selon Bosco, le Pascal de Ravaisson n'est pas le Pascal romantiquement déchiré, ni le sceptique de Cousin, mais plutôt un autre Pascal, «lucide et réconcilié, à la vie multiforme et aux fidélités et aux intérêts nombreux; un homme qui ne méprisait pas l'intelligence, mais qui lui proposait plutôt un exercice conscient dans les divers domaines; une âme vivant de cet amour unique qui le faisait (et le fait toujours!) conquérant : dans une « logique du cœur » renouvelée qui admettait des liens encore plus étroits que la religion et la philosophie ${ }^{19}$ ». En substance, Ravaisson nous offre un « Pascal non pas déchiré, un homme, au contraire, qui avait traversé de multiples expériences, proposant une philosophie à l'épreuve du réel et de la vie; et pour cette raison, avec le destin inscrit de la «philosophie du cœur ${ }^{20} » »$. Une philosophie qui, comme on le déduit des Pensées, rappelle et adresse les hommes au cœur, «non pas pour se complaire, mais pour libérer les énergies de l'amour ${ }^{21} »$.

\section{Le réalisme politique d'un croyant}

L'exigence de redécouvrir un Pascal plus attentif aux besoins pratiques de l'homme explique l'intérêt que recueille le penseur politique. Un aspect jusque-là peu exploré, certes, mais sur lequel aujourd'hui les chercheurs semblent pourtant apporter quelque 
lumière ; il suffit de penser que ces dernières années ont vu publier en Italie un grand nombre de travaux consacrés à Pascal penseur éthico-politique. De ces travaux émerge, dans l'ensemble, l'image d'un réaliste, observateur aigu des faits, conscient de l'impossibilité de créer une société juste, sans se montrer pour autant hostile à la politique. Pascal ne voit pas dans la politique une voie pour dessiner une société idéale, mais plutôt un moyen habile pour guider la société en prenant appui sur l'imagination, la coutume et la force.

L'ordre politique a évidemment son utilité propre : la politique, en fait, met un frein au désordre chaotique de la société, habituant l'homme au respect des lois. Habitude de l'obéissance aux lois qui, dans une "société avec Dieu », se transforme en obéissance à la volonté de Dieu, grâce à laquelle il est possible de créer un ordre juste, fondé sur la charité.

Dans ces nouveaux travaux italiens émerge un Pascal non plus "antipolitique ", qui se serait moqué de la politique au point de la faire coïncider avec la folie, avec une «tentative d'assistance psychiatrique, avec un pronostic thérapeutique limité à la perspective du moindre mal ${ }^{22}$ ", mais un Pascal «attentif à la «spécificité » du politique et aux nombreux aspects du politique moderne ${ }^{23} »$ : c'est sur lui que Domenico Bosco fait porter sa réflexion.

Ce critique, dans l'introduction aux Fragments politiques de Pascal, où il recueille une série d'extraits des Pensées, des Provinciales et des Trois discours sur la condition des Grands, présente un Pascal «réaliste» qui voit dans la politique non un moyen de perfectionnement de l'homme et du monde, mais plutôt un frein opposé au chaos et la dégénérescence de la société.

21 En somme, si d'un côté la politique n'est plus pour Pascal un degré pour engendrer une société juste, parce qu'elle est « sans la grâce et l'impossible charité ${ }^{24}$ », de l'autre côté, la politique demeure un instrument efficace pour guider la société, parce que «le propre de la puissance est de protége ${ }^{25} »$. Et si le proprium de la politique doit être celui de protéger la société, alors on comprend peut-être pourquoi Pascal, comme en témoigne Nicole, apparaît « très clairement favorable à l'importance de la politique ${ }^{26}$ ".

Que Pascal ait témoigné un intérêt certain pour la politique, en témoigne la conscience que seule une «bonne politique » est à même de maintenir la paix : le bien suprême, pour l'accomplissement duquel le penseur clermontois va jusqu'à justifier l'usage de la dissimulation, du mensonge autorisé.

Utiliser le mensonge comme un moyen pour parvenir à une fin, et caractériser la paix comme la fin qui justifie le moyen, n'autorise absolument pas à accuser Pascal de «réalisme cynique, quoique se guidant sur sa conscience ${ }^{27}$ ». Si faire de la «bonne politique " signifie certainement cacher la vérité au peuple, cela signifie aussi ne pas perdre de vue cette "pensées cachée » qui sert à éduquer « une classe de gens habiles à l'éthos sévère de leur propre responsabilitée ${ }^{8}$ ».

Chiara Piazzesi s'est attachée à l'importance et à la valeur pédagogique de la pensée cachée : elle considère la double pensée comme un aspect clé de la vision politique de Pascal. Cette double pensée est celle que Pascal recommande au jeune fils du duc de Luynes, qui, s'il veut être un "bon politique», ne doit pas oublier que sa condition comme celle du naufragé, jeté sur une île inconnue, et reconnu par les habitants comme leur roi - n'est pas fondé sur la supériorité naturelle d'un homme sur les autres, mais bien sur l'établissement humain. Il doit pourtant se comporter comme les hommes de son rang, 
respectant la convention et la croyance du peuple; c'est seulement ainsi qu'il peut effectivement maintenir la paix ${ }^{29}$.

La double pensée se présente, donc, comme une recommandation du devoir et l'expression de la liberté. En fait, comme pensée apparente, elle recommande de respecter les lois et les institutions par le maintien et l'ordre de la société ; mais comme pensée cachée, elle permet d'exprimer librement notre opinion sur la personne qui se présente à nous, indépendamment de la position sociale qu'elle occupe.

Si c'est un devoir d'institution de porter respect à un prince par le biais de formes extérieures comme le saluer, se tenir debout en sa présence, s'agenouiller, ce n'est pas un devoir de l'estimer pour son rôle social. Si ce prince est « honnête homme ", c'est-à-dire s'il est grand dans l'ordre des "grandeurs naturelles ", alors il est juste qu'on lui rende non seulement l'hommage extérieur, mais l'estime intérieure ; mais si ce prince n'est pas " honnête homme ", alors, à côté de l'hommage extérieur dû à son rang, il est juste qu'on doit lui réserve « le mépris intérieur ».

Cette prise de conscience, qui permet de distinguer le respect extérieur dû aux grandeurs d'institution du respect intérieur dû aux grandeurs naturelles, « doit rendre à l'homme sa liberté à l'égard des chaînes de la coutume, sans pour autant ébranler la puissance « extérieure $»^{30}$ ".

L'étude de $\mathrm{M}^{\mathrm{me}}$ Piazzesi s'arrête aussi sur le rôle central que la coutume, comprise comme approfondissement continu, et non comme simple répétition, trouve dans les dynamiques relationnelles et dans la définition de la géographie de l'espace social, et surtout par rapport au pouvoir ${ }^{31}$. Partant de l'interprétation de la philosophie de Pascal proposée par P. Bourdieu ${ }^{32}$, elle tente de « réactualiser ", la pensée anthropologique et sociologique du philosophe clermontois, à partir de sa réflexion sur le rôle social de la coutume et ses liaisons avec le pouvoir.

29 Pour Pascal, la coutume est «le liant social par excellence» qui, modelant les comportements, les modes et les relations entre les hommes, maintient ferme la stabilité de l'ordre social. La coutume n'est pas seulement indispensable au maintien d'une société, mais elle est juste "relativement à l'utilité sociale qu'elle réalise ${ }^{33}$ ». C'est en fait «l'instrument par le biais duquel il est possible de "domestiquer» le moi constitutivement injuste " en soi, parce qu'il se fait centre de tout ", donc « incommode aux autres, parce qu'il veut les asservir ", et par conséquent porteur d'une tendance centrifuge qui s'oppose à la cohésion sociale, à la société même et à l'institution d'un ordre. Orientant la perception et la pratique, la coutume fait que les divergences et les conflits - potentiellement universels - se trouvent réduits au minimum, tout comme l'espace pour une rediscussion du pouvoir; l'effet obtenu est la solidité du pouvoir institué et la stabilité de l'ordre social ${ }^{34}$ ».

30 La coutume, par conséquent, est liée indissolublement à l'ordre institué: c'est le fondement des lois et de la stabilité du pouvoir. Si à l'origine la société se forme par la force, qui divise la communauté sur la base des relations de pouvoir entre les forts et les faibles, les vainqueurs et les vaincus, par la suite la société se maintient par le moyen de la coutume, lorsque la force fait défaut. Grâce à la coutume, l'acteur social s'habitue à vivre en respectant un certain ordre, savoir en se comportant automatiquement comme si l'environnement dans lequel il vit était le seul possible.

31 C'est pourquoi la coutume réalise dans l'individu la fin de la société même, laquelle devient ce lieu d'activité, de divertissement, dont l'homme se sert pour oublier son propre 
néant. Mais l'homme peut se soustraire à ce jeu, à cette illusion à laquelle la coutume l'avait habitué, moyennant l'usage correct et constant de la raison et par suite de la double pensée.

Avec le recours à la raison, entendue comme unique antidote à la fausseté qui règle les pratiques sociales, Pascal avance « la plus grande des illusions scolastiques (...), celle de la pure pensée" qui conçoit la raison complètement indépendante à l'égard "des conditionnements que la vie relationnelle (...) exerce sur les personnes dans leur totalité35 ». Toutefois l'erreur de Pascal n'est une erreur qu'à moitié. S'il est vrai, en fait, qu'il voit dans la prise de conscience de la fausseté des relations sociales l'unique instrument pour se libérer de ces conditionnements, il est tout aussi vrai qu'il "n'induit pas le sujet pensant à se soustraire totalement aux dynamiques du pouvoir qui règlent les relations sociales, pouvoir qui doit être respecté et conservé intégralement pour maintenir intact l'ordre de la société ${ }^{36} »$.

Le recours à la double pensée, s'il dévoile la nullité du pouvoir en le vidant de sa valeur absolue et en montrant la relativité de la coutume, ne soustrait pas pour autant le sujet aux conditionnements sociaux auxquels il est soumis en tant qu'automate et en tant que raison.

Pour avoir une ample vision d'ensemble de la pensée politique pascalienne, il est nécessaire de renvoyer au travail de $\mathrm{F}$. Semerari ${ }^{37}$, qui, réfléchissant sur la divergence théorique entre Hobbes et Locke d'une part, et Pascal de l'autre, offre au lecteur une histoire panoramique de la controverse éthico-politique du XVII ${ }^{e}$ siècle, attirant particulièrement l'attention sur la délicate question du fondement du droit et de la justice. Si Hobbes et Locke se présentent comme ceux qui représentent le plus grand moment de vitalité et d'affirmation du jus naturalisme au XVII ${ }^{e}$ siècle, Pascal à l'inverse, exprime un moment d'une crise très particulière.

Hobbes et Locke sont les théoriciens d'un droit et d'une loi naturels susceptibles d'une réalisation historique ; Pascal au contraire nie non pas tant l'existence des lois naturelles, que la possibilité pour l'homme de se conformer à ces lois. A cause du péché, l'homme, qui vit dans la corruption de sa nature, ne peut en fait édifier une vraie justice. La justice des hommes est une "imagination", qui cependant doit être maintenue et respectée parce qu'elle seule peut maintenir l'ordre et la paix sociale. Pour Semerari, c'est ce qui explique la crise du jus naturalisme chez Pascal : à ce dernier manque la confiance que Hobbes et Locke gardent en la possibilité de faire coïncider lois naturelles et lois positives. Et, cela d'autant plus que, à la différence de Hobbes et de Locke, Pascal est convaincu que les principes que les hommes présentent souvent comme principes naturels, sont en réalité seulement des principes habituels, historiquement déterminés ${ }^{38}$.

Pascal néanmoins ne dissout pas la catégorie du naturel, mais il montre le manque de fondement des usages que les hommes en font. Dans le fond, lui-même est convaincu de la nécessité de donner aux lois une apparence de naturalité pour en garantir le respect, ainsi que le maintien de la paix. Le jus naturalisme devient ainsi pour Pascal «un instrument conscient de recherche de consensus ${ }^{39}$ ».

La divergence entre les trois philosophes devient encore plus aiguë, lorsqu'ils affrontent le problème de la revendication des mérites individuels contre le vieil établissement des privilèges sociaux. Devant les deux systèmes sociaux, celui du Moyen Âge fondé sur des privilèges présumés naturels, et celui de l'époque moderne fondé sur la reconnaissance des mérites individuels, Pascal prend une position bien précise : il est favorable au vieux 
système, parce que c'est le seul à offrir aux hommes cette sécurité et cette paix que le nouveau ne serait pas, lui, en mesure de garantir. Le système fondé sur les mérites, en fait, «par le principe même sur lequel il se fonde est destiné à donner lieu à la guerre civile ${ }^{40} »$.

Toutefois, cela n'ôte pas son importance au système moderne qui, en tout état de cause, se trouve intégré avec l'ancien: si en effet il y a des fonctions pour lesquelles vaut le critère ancien (comme le cas du choix de celui qui doit gouverner un État), il y en a d'autres où s'impose le critère de la capacité personnelle : pour diriger un navire, on ne cherche pas le passager qui est de la plus noble maison ${ }^{41}$.

Malgré quoi, Pascal apparaît à Semerari comme le défenseur sui generis d'un vieux système fondé sur les privilèges naturels, contre lequel, au-delà des différentes interprétations, Hobbes, Locke et Spinoza explorent la nécessité de construire un nouveau modèle d'organisation, fondé sur la reconnaissance de la capacité de l'individu ${ }^{42}$.

Dans cette perspective, le travail de Isabella Adinolfi, Le cercle brisé $e^{43}$, est digne d'attention: outre qu'il démontre que c'est sans fondement qu'on fait de Pascal un adversaire «cynique » du droit naturel et un défenseur du relativisme éthique, il offre une intéressante analyse de la politique pascalienne, où réalisme et utopisme se présentent comme deux volets rigoureusement complémentaires.

$41 \quad \mathrm{M}^{\mathrm{me}}$ Adinolfi prend pour point de départ les idées de certains critiques ${ }^{44}$ qui, se référant au fragment 94-60, et particulièrement au passage où Pascal semble nier ironiquement l'existence des lois naturelles ${ }^{45}$, ont assuré que Pascal «n'a rien de commun avec la théorie du droit naturel» et que, du principe que le hasard "a disséminé les lois humaines, il fait dériver l'inexistence les lois universelles ${ }^{46}$ ».

Chez Goldmann ce dernier jugement trouve une confirmation dans d'autres fragments des Pensées consacrés au rapport de la force avec la justice, où la force semble prendre le dessus sur la justice. L'analyse de ces fragments a conduit certains chercheurs à taxer Pascal tantôt de " cynisme ${ }^{47}$ ", tantôt « d'amoralisme politique », tantôt à l'accuser d'être un « défenseur de l'absolutisme brutal ${ }^{48}$ " ou de pencher pour un "relativisme à outrance ${ }^{49}$ ». Autant d'accusations qui - comme l'a justement observé Jean Mesnard - négligent que Pascal est simplement en train de constater les faits dans un état d'esprit réaliste, et non pas de définir ce qui est juste ${ }^{50}$.

De ce point de vue $\mathrm{M}^{\mathrm{me}}$ Adinolfi repousse aussi l'accusation d'un Pascal adversaire du droit naturel et défenseur du relativisme éthique ${ }^{51}$ : au terme d'une analyse exacte et pointilleuse, elle n'en trouve pas trace, même dans le très contesté fragment 94-60, où Pascal, tout en affirmant qu' " il y a sans doute des lois naturelles », ajoute tout de suite après que « cette belle raison corrompue a tout corrompu ». À cet endroit Pascal ne nie pas «l'universelle possibilité de connaître la loi morale, mais sa présence dans les lois positives, son application effective dans les œuvres des gouvernants, et son observation dans leurs actions et leurs intentions ${ }^{52}$ ». Il « ne nie pas la connaissance du principe de ce qui est juste, mais il nie que cette connaissance soit « suivie ». La corruption, en d'autres termes, regarderait surtout la volonté de l'homme ${ }^{53} »$.

Si l'existence des lois naturelles se trouve, pour ainsi dire, «cachée » par une raison, qui dans le cadre politique ressent avec plus de force l'influence d'une volonté corrompue et intéressée, comment alors l'homme pourrait-il connaître la justice ? La réponse, selon $\mathrm{M}$ me Adinolfi, doit être cherchée dans la distinction pascalienne entre raison et cœur ; ce dernier en fait est l'organe qui, conservant les traces d'une nature incorrompue, 
témoigne que dans le fond le péché n'a pas corrompu complètement la capacité de l'homme de connaître le vrai et le bien, et d'apercevoir la vraie morale.

Nous sommes donc loin de ceux qui taxent Pascal de relativisme éthique et de scepticisme anthropologique ${ }^{54}$; et nous sommes également éloignés de le considérer comme un adversaire du droit naturel. S'il est vrai, d'un côté, que dans le célèbre fragment 94-60, fragment mal interprété par ceux qui ont cherché à « dissimuler » un possible rapport entre Pascal et le droit, il nie la réalité d'une loi universelle, de l'autre côté, il est tout aussi vrai que en un autre endroit il retrouve une telle loi dans celle qui est «cette maxime, la plus générale de toutes celles qui sont parmi les hommes, que chacun suive les mœurs de son pays ». En somme, personne ne peut douter du fait qu'en tout lieu existe la loi qui impose d'observer les lois. Cela ne signifie-t-il pas que Pascal, dans le fragment 94, pose les bases de l'existence de la loi naturelle, qui consisterait dans l'obéissance aux lois positives?

Il y a trois attitudes décrites par Pascal à l'égard de l'ordre politique $: 1$. celle de l'homme ordinaire qui suit la loi de son propre pays parce qu'il la croit juste, adhérant ainsi à une «justice imaginaire»; 2 . celle du demi-habile ou du demi-savant qui, se comportant de manière diamétralement opposée à celle de l'homme ordinaire, pousse le peuple à la révolution, en lui montrant l'injustice des lois; 3. l'attitude du sage, qui au contraire recourt au «mensonge politique », s'abstenant de révéler au peuple l'injustice des lois, afin de maintenir la paix.

Maintenant, ce dernier comportement, s'il semble le plus conforme à la nature de l'homme, n'en est pas pour autant celui que préfère Pascal, comme l'ont pensé des critiques comme Maritain et Auerbach. Au-dessus de ces trois comportements, en fait, il en existe deux autres : celui du chrétien dévot zélé, et celui du chrétien parfait ${ }^{55}$.

Le "chrétien dévot», comme le demi-savant, méprise les grands, quoique pour un autre motif : il pense que tous les hommes sont libres et égaux devant Dieu. Il confond - et là est l'erreur ! - l'ordre de la chair et l'ordre de la charité, et oublie les conséquences du péché originel, qu'au contraire le chrétien parfait a toujours devant les yeux. Ce dernier, au contraire de l'habile, n'est pas disposé à tromper le peuple, même pour son bien. Le chrétien parfait, sachant bien combien il est dangereux de dire au peuple que les lois ne sont pas justes, ne lui dissimule pas la vérité, mais lui demande d'obéir aux lois parce qu'elles sont lois, comme l'on obéit aux supérieurs non parce qu'ils sont justes, mais parce qu'ils sont supérieurs ${ }^{56}$.

49 Le mensonge, fût-il autorisé, n'appartient donc pas au chrétien, pour lequel la fin ne justifie pas les moyens. Voilà pourquoi, si les vrais chrétiens s'assujettissent à la folie du monde, ils ne le font pas par respect de la folie, mais par égard à l'ordre de Dieu, qui pour punir les hommes les a assujettis à cette folie ${ }^{57}$.

50 Mais au fond, toutes les lois auxquelles le chrétien se soumet ne sont pas folles : c'est le cas des lois contre l'homicide, des lois contre le vol et beaucoup d'autres lois en l'absence desquelles, si elles n'existaient pas, le pouvoir même s'écroulerait. Par conséquent, même si l'obéissance aux lois peut revêtir pour un chrétien le caractère du compromis, puisqu'elles ne sont pas toutes justes, cela ne signifie pas pour autant que l'obéissance du chrétien ne soit, comme le soutiennent Auerbach et Maritain, qu'une soumission résignée aux grands de ce monde. En fait, si une loi contredisait la véritable justice, alors, pour Pascal, le chrétien aurait le devoir de ne pas la respecter. Et dans tout cela il n'y a pas de contradiction: dans les deux cas, - dans le respect des lois ou dans le refus de les 
respecter - on obéit à la volonté divine qui, dans le premier cas, se présente comme dessein providentiel dans les événements historiques, et, dans le second, révèle un appel personnel à s'opposer et à résister au mal ${ }^{58}$.

51 En définitive, comme l'a montré Domenico Bosco, Pascal caractérise les perspectives d'une politique possible et bonne ${ }^{59}$, qui peut coexister avec l'attitude du chrétien, lequel, lorsqu'il agit aussi à l'intérieur de «l'ordre des corps » ne doit pas perdre de vue «l'ordre de la charité ", dont il tire l'inspiration pour agir non selon sa propre volonté, mais suivant la volonté de Dieu.

52 Analysant le devoir du « chrétien parfait » à l'intérieur de la société, $\mathrm{M}^{\mathrm{me}}$ Adinolfi déplace l'attention sur cette complémentarité paradoxale, mais non pas impossible, entre le réalisme et l'utopisme présents dans la politique pascalienne. Dans une «société sans Dieu » où les rois, quoique chrétiens, considèrent la loi de Dieu sur un mode extérieur et formel, le "chrétien parfait », pour éviter de confondre l'ordre des corps et celui de la charité, peut seulement se soumettre à la « folie du monde ». Et c'est proprement cela qui est le ferme réalisme de Pascal.

Mais dans une "société avec Dieu», le chrétien parfait, conscient de ce que la vraie justice ne naît pas de l'extérieur, mais bien de l'intérieur moyennant la conversion du cœur, peut se servir de son témoignage pour « convaincre les hommes d'entreprendre le pénible chemin de la conversion »; un devoir extrêmement délicat qui exige une discipline difficile et une obéissance prompte et totale.

C'est en cela le pur utopisme de Pascal ${ }^{60}$. Requérir une soumission totale à l'égard de la volonté de Dieu signifie renoncer à l'amour de soi et se rendre libre moyennant « la loi de l'amour ou l'amour de la loi ». Et c'est là la « république chrétienne » indiquée par Pascal, une communauté qui a «Dieu seul pour seigneur» et «deux commandements seulement »: l'amour pour Dieu et l'amour pour le prochain, grâce auxquels il est possible de construire un ordre authentique et juste. Et, grâce à l'amour, "l'ordo concupiscentiae » se transforme en « ordo caritatis ${ }^{61} »$.

\section{Le besoin de l'éthique}

Le fait que l'intérêt pour Pascal se soit réveillé aujourd'hui en Italie semble être lié à la prise de conscience que le penseur français avait encore quelque chose à dire et que sa philosophie n'est pas, comme le veut Del Noce, destinée à être dépassée. À ce propos, Claudio Ciancio se rattache à Del Noce, dans la lecture d'un Pascal dans lequel «le plus radical cartésianisme coexiste avec le plus radical anticartésianisme ${ }^{62}$ ", c'est-à-dire d'un Pascal qui représente le début de la pensée moderne avec un anticartésianisme qui prend assise sur des présupposés cartésiens. Mais Ciancio se sépare de Del Noce, lorsque ce dernier considère la pensée de Pascal comme le simple commencement d'une lignée qui déboucherait sur l'ontologisme. De ce point de vue on peut affirmer que Pascal offre une pensée «consistante, autosuffisante et particulièrement actuelle ${ }^{63}$ ", et qui, en confrontation avec Descartes, « en ce qu'il sait porter en lumière les tensions de fond qui parcourent la modernité et la constituent encore, en exprime bien mieux l'essence, ce qui rend sa pensée est beaucoup plus actuelle ${ }^{64} »$.

Giuseppe Brescia est un défenseur convaincu de l'actualité de la pensée pascalienne. En effet, en une époque déchirée par la crise intellectuelle et morale, qui envoie des signaux toujours plus alarmants, et qui ne parvient pas à trouver une issue aux actuels problèmes 
existentiels, écologiques et bioéthiques, Pascal semble vraiment s'être remis à jeter des traits de lumière ${ }^{65} \%$.

«Ne t'inquiète donc pas, écrit le philosophe clermontois. Tout nous peut être mortel, même les choses faites pour nous servir, comme dans la nature les murailles peuvent nous tuer et les degrés nous tuer si nous n'allons avec justesse. Le moindre mouvement importe à toute la nature, la mer entière change pour une pierre. Ainsi dans la grâce la moindre action importe pour ses suites à tout; donc tout est important. En chaque action il faut regarder outre l'action, à notre état présent, passé, futur et des autres à quoi elle importe. Et voir les liaisons de toutes ces choses et lors on sera bien retenu ${ }^{66}$ ». C'est cela le conseil de Pascal à l'homme d'aujourd'hui, écrasé par une technologie déferlante et en proie à la folie de la volonté de puissance : la prudence; la "prudence comme vertu éthique »; la " prudence comme synthèse des états d'âme ».

À la prudence caractérisée par Brescia, Bernardo Razzotti fait succéder un autre conseil pascalien, plus incisif que le premier, à l'intention d'un homme désormais enivré par l'optimisme rationaliste et dénué de valeurs: «N'oubliez pas avant tout que l'homme est un être religieux ${ }^{67} »$.

Et la signification de l'être religieux équivaut à vivre la relation féconde de l'unité que l'homme doit réaliser avant tout en lui-même, et se diriger vers « cet Être exemplaire et secret » à travers la «profondeur des raisons du cœur. Cette "philosophie » convainc, remplit déjà le monde ${ }^{68}$ ", une philosophie qui a «fouillé à l'intérieur de l'homme, qui est celle de toujours", "qui a su convoquer l'âme humaine vers les problèmes de l'introspection, réveillant le sens du divin et de l'invisible, alors que justement cette âme même semblait toucher le fond de la désespérance et de l'ignorance face au problème du mal et de la mort ${ }^{69} »$. Voilà un motif qui convainc de l'actualité du penseur français, à telle enseigne que " quand Pascal a écrit hier, il aurait pu écrire aujourd'hui ${ }^{70}$ ».

De là l'invite à réfléchir sur la Préface au traité du vide, sur l'importance de laquelle insistent de manière particulière aussi bien Pareyson ${ }^{71}$ que Razzotti. Celle-ci en fait est l'expression la plus haute de la modernité et de l'actualité de Pascal qui, récusant la "seule rationalité scientifique» et la seule "mystique religieuse ${ }^{72}$ ", indique, avec l'équilibre qui le caractérise et qui lui confère son autorité, la voie la plus juste pour ne pas se faire emporter par un progrès scientifique et technologique toujours plus inquiétant, et ne pas céder aux illusions de la superstition et de l'intégrisme.

61 L'équilibre entre la religion et la raison, esquissé dans la Préface, et la dénonciation de la déraison de qui ne reconnaît pas un tel équilibre sont, selon Razzotti, les deux moments fondamentaux du dessein apologétique de Pascal. Un dessein propre à interpréter la religion et son message avec l'emploi des catégories philosophiques, qui permettent de récupérer la singularité, le caractère concret, la liberté et la dimension dialogique de l'être humain, entendu comme un être non pas fermé, mais essentiellement ouvert à la rencontre avec d'autres personnes ${ }^{73}$.

Partant de cette interprétation "originale », Razzotti fait de Pascal « un précurseur du personnalisme ${ }^{74} »$. Cette lecture est en mesure, d'un côté, de recueillir l'étroit lien qui unit Dieu à l'homme et l'homme à Dieu; et de l'autre, de permettre de dépasser «l'historicisme positiviste», «le subjectivisme transcendental», «la vision exclusivement existentielle du message chrétien », et «la réduction révolutionnaire de l'Évangile ${ }^{75} »$. Nous sommes donc très loin de cette image d'un Pascal tragique qui voit dans l'homme un être tourmenté, pris entre l'ennui, l'inquiétude et le divertissement ${ }^{76}$. 
Si la conciliation pascalienne entre science et religion a porté Razzotti à faire de Pascal un précurseur du personnalisme, Pareyson tire de cette conciliation, ou mieux de cette collaboration entre science et religion, deux conséquences importantes pour la morale : « la première est que l'idée de l'homme progresse dans la science, mais non dans la morale; la seconde est que la science, valide en elle-même, est inutile à la morale ${ }^{77}$ ".

4 Ces conséquences naissent toutes deux du concept que l'homme est « fait seulement pour l'infini "; un concept qui, selon que l'on envisage le terrain scientifique ou celui de la morale, engendre deux effets opposés. Dans le premier cas, il se joint à une maturation de l'homme qui manifeste la fécondité de son intelligence particulièrement dans l'augmentation continuelle et illimitée des connaissances scientifiques ; dans le second, la morale, cette tendance à l'infini qui anime l'homme, toujours insatisfait, à dissiper sa vie en passant d'un désir à un autre, engendre au contraire une «dissipation illimitée, un perpétuel état d'enfance et de minorité ${ }^{78}$ ".

5 Si les affirmations de Pareyson paraissent acceptables, lorsque, mettant en évidence la collaboration étroite entre la science et la religion, il semble s'éloigner de ceux qui ont voulu voir dans Pascal soit le savant, soit le mystique, il apparaît cependant convenable de préciser le concept « d'inutilité » de la science dans le domaine moral. Il n'y a pas de doute que la science n'a aucune utilité dans le domaine moral, que ses démonstrations sont inutiles pour expliquer le mystère de l'homme et pour lui indiquer un possible chemin de salut; mais à mon sens ${ }^{79}$, on ne doit pas oublier que, grâce à la réflexion scientifique aussi, et par conséquent à l'application de la raison dans le domaine de la science abstraite, l'homme se rend compte qu'il existe « des réflexions qui valent mieux que tout le reste de la géométrie », réflexions qui ne peuvent certainement naître de la science, et que par conséquent doivent être cherchées d'un autre côté.

66 C'est dans cette affirmation que se trouve la "solution ", si l'on peut parler de solution, de la coexistence possible de la science et de la morale. Si Pascal, mathématicien, physicien, ingénieur prestigieux, engage d'un côté avec autorité et équilibre à " réfléchir sur des réflexions qui valent beaucoup plus que la science en elle-même ", de l'autre côté il semble exhorter l'homme moral à indiquer à l'homme de science, sans mettre en discussion l'autonomie et la liberté de la science, ces points fixes dont, aujourd'hui, les traces semblent perdues, que ce soit dans le champ scientifique ou dans le champ éthique ${ }^{80}$.

La collaboration entre la science et la religion, soutenue avec force par Pascal, porte donc à la reconnaissance du primat de l'éthique, sûrement chrétienne, point sur lequel insistent, entre autres, Pareyson et Peratoner. Cette éthique de Pascal se trouve en une position «supérieure » par rapport à la science, parce qu'elle agit, ou au moins devrait agir, dans l'ordre supérieur à celui de l'esprit, l'ordre surnaturel de la charité.

6 Le fait que l'éthique agisse, ou devrait agir, dans l'ordre surnaturel de la charité, explique, selon Pareyson, l'impossibilité de construire une morale philosophique. Avec la raison philosophique, il ne semble pas possible de construire une éthique universelle, parce que la raison est incapable de démontrer l'existence ou l'inexistence de ce bien suprême, dont la fin ultime est Dieu, d'où « tirer la norme de notre conduite ${ }^{81}$ ", et affirmer l'immortalité de l'âme.

69 La construction d'une morale dépend, par conséquent, uniquement de la découverte de ce bien suprême, principe et fin de l'homme : «Dieu est donc le fondement de la morale, et il importe surtout, en vue de la fondation d'une morale, d'établir l'existence de Dieu ${ }^{82} »$. Ce 
problème de l'existence de Dieu n'est pas spéculatif, parce que de son existence ou de sa non-existence dépend toute l'action humaine, parce qu'il n'est pas seulement l'objet de la connaissance, mais aussi d'amour : connaitre Dieu est le regarder comme norme d'action ; le posséder est être heureux.

À la lumière de cette importance pratique de l'existence de Dieu on doit lire, selon moi, le fameux fragment du pari, où Pascal invite l'interlocuteur athée à parier sur la possible existence de Dieu, en lui indiquant l'utilité pratique, en rapport avec le bonheur, qui proviendrait de cette option.

71 Mais le fragment du pari présente aussi une importance spéculative, si minime soit-elle. Ce qui ne veut certainement pas dire qu'il constitue une preuve rationnelle de l'existence de Dieu ; toutefois il est impossible de nier que dans le raisonnement du pari se trouve une tentative pour porter l'incrédule à traiter rationnellement de l'existence de Dieu comme hypothèse possible $e^{83}$.

De la réflexion pascalienne sur l'infini et son incommensurabilité à l'égard du fini apparaissent clairement les limites de notre raison, laquelle s'avère incapable de saisir l'infini divin. De là le « choix » de s'accorder au cœur, dont la connaissance évidente des principes premiers offre à l'activité rationnelle la possibilité, même indirecte, d'admettre l'existence de l'infini mathématique.

73 Mais dans cette démarche, notre raison peut regarder au-delà, et «supposer " directement, sur la base de l'existence de l'infini mathématique, l'existence d'un infini divin dans lequel tous les infinis ont leur origine. En se fondant sur cette démarche, Pascal se porte donc à affirmer sur une assise rationnelle, comme hypothèse possible, l'existence de Dieu.

74 Même à vouloir accepter la thèse de Pareyson, selon lequel cet infini spatial, temporel, numérique dont nous connaissons l'existence et non la nature, est un infini négatif, savoir l'indéfini, «plus précisément l'illimité dans l'espace, dans le temps, dans le nombre : cela qui est indéfiniment innombrable ou parce que étendu et divisible, ou parce que durable et multipliable ${ }^{84}$ ", un infini par conséquent numérique duquel la raison réussit à saisir l'existence, même à vouloir accepter une telle thèse, on ne parvient pas complètement à nier ce qui a été dit un peu plus haut.

75 En somme, à mon sens, la reconnaissance de l'existence de l'infini numérique, même si elle est négative, est la reconnaissance de la potentialité inscrite dans notre raison de s'élever jusqu'à l'infini, ascension qui à coup sûr ne lui fera pas rejoindre l'infini divin, mais qui est malgré tout utile pour pouvoir réfléchir sur la possible existence d'un infini substantiel, dont l'infini numérique peut avoir tiré son origine ${ }^{85}$.

Si, pour parler en termes pascaliens, notre raison ne peut ni connaître ni démontrer Dieu, elle connait et « règle » cet ordre naturel que la nature a imprimé en toute chose, et possède en soi les instruments adaptés pour pouvoir admettre, au moins comme hypothèse possible, l'existence de Dieu, et, par conséquent, pouvoir « parier » en faveur de cet infini absolu et divin qui ordonne tout.

Mais de ce «pari » l'homme ne recevra pas la certitude de Dieu. Pour y parvenir, il est nécessaire qu'il abandonne l'esprit de géométrie, afin de laisser place à l'esprit de finesse; il faut abandonner l'ordre naturel de la raison, pour se rapprocher de l'ordre surnaturel de la charité qui dévoile la nature et l'existence de l'infini absolu. 
78 Au terme de l'analyse pascalienne sur l'infini et son rapport à l'homme, notre raison ne paraît donc pas si impuissante : sa faiblesse ne peut en fait être considérée telle que si on la compare à l'activité cognitive du cœur qui connaît l'infini directement. Sans doute la raison humaine ne connaît pas directement ; toutefois les principes évidents qu'elle reçoit du cœur - principes qu'elle ne peut démontrer - ont leur importance. Les reconnaissant, la raison s'habitue en fait à s'ouvrir aux orientations du cœur, qui reste toujours l'unique instrument qui permet de saisir l'infini divin.

79 À ce propos apparaît opportune la réflexion d'Alberto Peratoner, selon lequel la nécessité de rechercher le vrai bien au-delà de l'usage spéculatif de la raison scientifique, dans l'ordre de la charité, ne semble pas exclure l'usage correct du bien penser qui constitue une étape nécessaire dans le procès d'élévation de l'ordre de l'esprit à l'ordre surnaturel de la charité. Aussi Peratoner souligne-t-il en conséquence l'importance de l'opuscule De l'esprit géométrique, dans lequel il est dit que l'usage correct de la méthode géométrique conduit à des « réflexions qui valent beaucoup mieux que la géométrie elle-même ». Il y a dans cette phrase l'affirmation du primat de l'éthique sur la science, qui « reste une constante de la pensée de Pascal, qui dominera le terrain durant la polémique des Lettres Provinciales et de leur suite de factums des Écrits des curés de Paris, qui demeure fixée dans l'expression qui est presque une sentence : Notre cause est la cause de la morale chrétienne ${ }^{86}$ $»$.

Le problème de la construction d'une morale semble trouver sa solution propre dans le cœur. Mais la raison, quoique incapable d'argumenter exhaustivement sur le destin de l'homme et l'existence de Dieu - les deux piliers sur lesquels s'édifie la morale -, réalisent toutefois son devoir en rapport avec une morale indubitablement fondée sur la religion chrétienne. En fait, suivant ma thèse ${ }^{87}$, notre raison, sur la base de ces principes évidents qui proviennent du cœur, s'habitue et habitue à s'ouvrir aux orientations du cœur, favorisant ainsi l'édification de la « morale du cœur » sur laquelle insiste Pareyson.

81 Pour ce critique la vraie morale n'est pas celle de l'intellect, mais celle du cœur, de l'instinct, du sentiment. Admettre l'existence d'une morale de l'intellect signifierait, en fait, prétendre faire progresser la morale démonstrativement comme la science, en présupposant des principes dont seraient déduits des commandements. Mais cela est impossible, en premier lieu parce que la raison ne possède pas de principes, sinon par voie indirecte ; et en second lieu, parce que dans le champ de la morale, on ne peut rien démontrer.

82 Il ne reste donc que la morale du cœur, qui ne déduit pas les préceptes moraux de principes généraux, mais fait appel au jugement de la conscience qui juge à partir d'un principe unique « inspirateur de la morale » : l'amour de Dieu. "Chaque précepte, écrit Pareyson, doit être reconduit à la fin ultime, en chaque précepte doit être montrée la présence de la fin ultime, même tout précepte doit venir personnellement et dérivé chacun à son tour de l'unique inspiration morale qui est l'amour de Dieu ${ }^{88} »$. Et plus loin : « les cas particuliers, c'est le cœur qui les décide personnellement selon la conscience propre conforme à l'amour de Dieu ${ }^{89} "$.

83 À la lumière de tout cela, il apparaît évident que pour Pareyson la fondation de la morale est essentiellement religieuse. Mais si la morale est fondamentalement religieuse, et si la religion dépend de la foi, laquelle est un don exclusif de Dieu, devons-nous pour autant conclure que la possibilité de construire une morale soit barrée à l'homme privé du don de la foi? 
" parvient à la morale révélée comme l'unique port en mesure de garantir l'objectivité et la capacité de réalisation dans l'exercice de la pratique, en vertu de la grâce ${ }^{93}$ ", car, comme l'affirme Pascal, "l'homme n'est qu'un sujet plein d'erreur naturelle, et ineffaçable sans la grâce ${ }^{94} »$.

91 Si l'homme doit par conséquent viser à une éthique objective, alors celle-ci ne peut être que l'éthique chrétienne, «qui, dans la mesure où elle est révélée, trouve la racine et la raison de son objectivité dans le fait qu'elle vient directement de Dieu, mais comporte en même temps une origine de ses principes en une autre source que celle de la seule conscience individuelle ${ }^{95} »$. Dans la révélation, par conséquent, se trouve ce point fixe dont on a besoin, pour régler de manière objective la pratique propre, et libérer la morale 
de la dispersion relativiste : "Considérer Jésus-Christ en toutes les personnes, et nousmêmes ${ }^{96}$ ».

C'est là l'enseignement de Pascal, un penseur qui a été simultanément un grand savant et un grand croyant. Deux caractères qui lui donnent cette autorité nécessaire pour affirmer, devant l'homme savant et l'homme moral, le primat de l'éthique du cœur. Ce type d'éthique est la clé de la solution, parce que le cœur, possédé par tous les hommes indistinctement et naturellement par cet «instinct de s'élever ", a en soi la puissance d'accéder au vrai bien. Une puissance qui doit être alimentée avec la persévérance de la «bonne conduite » et renforcée par cet amour de Dieu dont seule le cœur est en mesure de s'enivrer. Et ce Dieu ne peut être le Dieu des philosophes, de la raison cartésienne, mais le Dieu d'Abraham, d'Isaac et de Jacob, le Dieu du christianisme, l'unique Dieu qui, se faisant homme en la personne de Jésus-Christ, sait comment parler au cœur de l'homme, dans la mesure où il en connaît la faiblesse et la misère. C'est en lui que se trouve donc la voie du salut, de la vérité, de la félicité et de la morale.

93 "J'admire ", écrit Pascal, une première et auguste religion, toute divine dans son autorité, dans sa durée, dans sa perpétuité, dans sa morale, dans sa conduite, dans sa doctrine, dans ses effets et ainsi je tends les bras à mon Libérateur, qui ayant été prédit durant 4000 ans est venu souffrir et mourir pour moi sur la terre dans les temps et dans toutes les circonstances qui en ont été prédites, et par sa grâce j'attends la mort en paix dans l'espérance de lui être éternellement uni et je vis cependant avec joie, soit dans les biens qu'il lui plaît de me donner, soit dans les maux qu'il m'envoie pour mon bien et qu'il m'a appris à souffrir par son exemple ${ }^{97} »$.

Et puis : " (J'aime tous les hommes comme mes frères, parce qu'ils sont tous rachetés.) J'aime la pauvreté parce qu'il l'a aimée. J'aime les biens parce qu'ils me donnent le moyen d'en assister les misérables. Je garde fidélité à tout le monde. Je ne rends point le mal à ceux qui m'en font, mais je leur souhaite une condition pareille à la mienne où l'on ne reçoit point de mal ni de bien de la part des hommes. J'essaye d'être juste, véritable, sincère et fidèle à tous les hommes et j'ai une tendresse de cœur pour ceux à qui Dieu m'a uni plus étroitement. Et soit que je sois seul ou à la vue des hommes j'ai en toutes mes actions la vue de Dieu, qui les doit juger et à qui je les ai toutes consacrées. Voilà quels sont mes sentiments. Et je bénis tous les jours de ma vie mon Rédempteur qui les a mis en moi et qui d'un homme plein de faiblesse, de misère, de concupiscence, d'orgueil et d'ambition a fait un homme exempt de tous ces maux par la force de la grâce, à laquelle toute la gloire en est due, n'ayant de moi que la misère et l'erreur ${ }^{98}$ ".

Dans ces deux fragments il est possible de distinguer, à mon sens, cette « règle » d'une " saine philosophie " grâce à laquelle le penseur clermontois semble indiquer aujourd'hui une voie alternative au nihilisme et au relativisme éthique. Un motif, qui dévoile peutêtre la raison pour laquelle nous nous sentons aujourd'hui encore liés à lui, pourquoi nous le considérons comme un penseur non de mode, qui répond au goût superficiel du moment, mais un penseur « actuel » qui reste encore substantiellement valide dans notre présent et pour lui.

96 À ce sujet, il est bon de rappeler en conclusion les paroles que Giuseppe Pezzino a récemment écrites pour présenter le "phénomène Pascal » aujourd'hui en Italie : « Loin de nous l'idée de vouloir tourner en arrière, pour idolâtrer un penseur momifié. Nous savons bien que, comme tout penseur, Pascal a ses racines historiques et présente ses ombres. En d'autres termes, il n'étonne et ne paralyse pas ceux qui entendent se mesurer de manière critique avec les idées encore vives et actuelles d'un penseur qui soit être 
compris historiquement et dépassé dialectiquement, pour faire face aux sollicitations et aux problèmes de notre présent. En somme, Pascal est un « classique » non parce qu'il est parfait, mais parce que, dépassant les critiques et les injures du temps, il ne cesse pas de parler de choses vivantes et passionnantes ${ }^{99} "$.

\section{NOTES}

1. L. Goldmann, Le dieu caché. Étude sur la vision tragique dans les Pensées de Pascal et dans le théâtre de Racine, Paris, Gallimard, 1955.

2. A. Del Noce, «Intorno all" antiumanesimo" di Pascal », in "Archivio di Filosofia", Padova, 1962, pp. 41-65.

3. A. Peratoner, Blaise Pascal. Ragione, rivelazione e fondazione dell'Etica. Il percorso dell'Apologie, Venezia, Cafoscarina, 2002, vol. 2.

4. A. Peratoner, "Oltre il frammento. Ragione architettonica delle "Pensées ", in L'incerto potere della ragione, a cura di G. Pezzino, Catania, CUECM, 2005, p. 62.

5. Ibid., p. 107.

6. B. Papasogli, «Introduzione » a B. Pascal, Pensieri, nuova edizione a cura di Ph. Sellier secondo l'« ordine » pascaliano, introduzione e traduzione di B. Papasogli, Roma, Città Nuova, 2003.

7. Ibid., p. 12.

8. F. P. Adorno, La ragione ordinata. Saggio su Pascal, Napoli, La Città del Sole, 2000.

9. Ibid. p. 154 .

10. L. Pezza, Le tentazioni del finito. Saggio su Pascal, Napoli, ESI, 2004, p. 31.

11. Ibid., p. 186.

12. F. P. Adorno, La ragione ordinata, cit., p. 164.

13. M V. Romeo, Verità e bene. Saggio su Pascal, Catania, CUECM, 2003 ; et aussi M. V. Romeo, Il numero e l'infinito. L'itinerario pascaliano dalla scienza alla filosofia, Catania, CUECM, 2004.

14. B. Pascal, Scritti matematici, riordinamento, traduzione e nota critico-bibliografica a cura di G. Brescia, Fasano, Schena, 1991.

15. G. Brescia, Pascal e l'ermeneutica, Fasano, Schena, 1989, p. 44.

16. « Les « raisons du cœur », écrit Brescia, la polémique contre le cartésianisme et le scientisme, le dramatique appel de la conscience aux valeurs du Christianisme ne sont pas en contraste, mais en rapport concret, (...) et en cohérence définitive avec la «découverte» du calcul des probabilités, les thèmes de l'engagement et le principe du pari et la possibilité même de la recherche scientifique, parce que le travail de la conscience morale est le préambule et la voie de la recherche » (G. Brescia, Pascal e l'ermeneutica, cit., p. 12).

17. B. Razzotti, Pascal e i suoi problemi. Riflessioni filosofiche ed etico-politiche, Lanciano, Carabba, 2004.

18. Ibid., p. 5.

19. D. Bosco, Un Pascal che ognuno avrebbe voluto scrivere, saggio introduttivo a $\mathrm{F}$. Ravaisson, $\mathrm{La}$ filosofia di Pascal, a cura di D. Bosco, Brescia, Morcelliana, 2003, p. 10.

20. Ibid., p. 39.

21. Ibid., p. 64.

22. E. Rossi, La politica come follia. Ironia e verità di Pascal, Roma, Studium, 1984, p. 19. 
23. D. Bosco, «Sfumati pascaliani », prefazione a B. Pascal, Frammenti politici, Brescia, Morcelliana, 2000 , p. 51.

24. Ibid., p. 13.

25. B. Pascal, Pensieri, edizione con testo a fronte a cura di C. Carena, Torino, Einaudi, 2004, Fr. 652-797. Dans les citations des fragments des Pensées, le premier numéro renvoie à l'édition de $\mathrm{C}$. Carena, le second à l'édition Lafuma.

26. Voir D. Bosco, «Sfumati pascaliani », cit., p. 37.

27. E. Rossi, La politica come follia. Ironia e verità di Pascal, cit., pp. 54-55.

28. R. Guardini, Pascal, Brescia, Morcelliana, 1956, p. 122.

29. Voir C. Piazzesi, Abitudine e potere. Da Pascal a Bourdieu, Pisa, ETS, 2003, pp. 60-1.

30. Ibid., p. 66.

31. Ibid., p. 17.

32. P. Bourdieu, Meditazioni pascaliane, traduzione italiana di A. Serra, Milano, Feltrinelli, 1998.

33. C. Piazzesi, Abitudine e potere. Da Pascal a Bourdieu, cit., p. 49.

34. Ibid., pp. 52-3.

35. Ibid., p. 115.

36. Ibid., p. 119.

37. F. Semerari, Potenza come diritto. Hobbes, Locke e Pascal, Bari, Dedalo, 1992.

38. Ibid., p. 134.

39. Ibid., p. 136.

40. Ibid., p. 141.

41. Ibid., p. 142.

42. Ibid., p. 220.

43. I. Adinolfi, Il cerchio spezzato, Roma, Città Nuova, 2000.

44. Ceux auxquels $\mathrm{M}^{\mathrm{me}}$ Adinolfi fait particulièrement référence sont $\mathrm{E}$. Auerbach, « Sulla teoria politica di Pascal », in Da Montaigne a Proust, traduzione italiana di G. Alberti, A. Carpi e V. Ruberl, Bari, De Donato, 1970, p. 143 et 128 ; et Goldmann, Le dieu caché, cit., p. 217 et 304.

45. «Ils confessent que la justice n'est pas dans ces coutumes, mais qu'elle réside dans les lois naturelles communes en tout pays. Certainement ils le soutiendraient opiniâtrement si la témérité du hasard qui a semé les lois humaines en avait rencontré au moins une qui fût universelle. Mais la plaisanterie est telle que le caprice des hommes s'est si bien diversifié qu'il n'y en a point. » (Fr. 94-60).

46. C. Ventimiglia, Società, politica, diritto : il cristiano e il mondo in Pascal e Domat, Parma, Zara, 1983, p. 118.

47. $\mathrm{M}^{\text {me }}$ Adinolfi se réfère encore à E. Auerbach, « Sulla teoria politica di Pascal », cit., p. 92 ; et à J. Maritain, «Les idées politiques de Pascal », in Jacques et Raïssa Maritain, CEuvres complètes, III, édition publiée par le Cercle d'études Jacques et Raïssa Maritain, Paris, Éditions universitairesÉdition Saint-Paul, Fribourg (Suisse), 1984, p. 185-192.

48. Voir I. Adinolfi, Il cerchio spezzato, cit., p. 93.

49. E. Rossi, La politica come follia. Ironia e verità di Pascal, cit., p. 68.

50. J. Mesnard, Les Pensées de Pascal, Paris, S.E.D.E.S., 1993, p. 205.

51. C. Ventimiglia, Società, politica, diritto : il cristiano e il mondo in Pascal e Domat, cit., p. 114.

52. I. Adinolfi, Il cerchio spezzato, cit., p. 94.

53. Ibid., pp. 95-6.

54. C. Ventimiglia, Società, politica, diritto : il cristiano e il mondo in Pascal e Domat, cit., p. 114.

55. « Raison des effets. Gradation. Le peuple honore les personnes de grande naissance, les demihabiles les méprisent disant que la naissance n'est pas un avantage de la personne mais du hasard. Les habiles les honorent, non par la pensée du peuple mais par la pensée de derrière. Les dévots qui ont plus de zèle que de science les méprisent malgré cette considération qui les fait honorer par les habiles, parce qu'ils en jugent par une nouvelle lumière que la piété leur donne, 
mais les chrétiens parfaits les honorent par une autre lumière supérieure. Ainsi se vont les opinions succédant du pour au contre selon qu'on a de lumière. » (Fr.124-90)

56. «Il est dangereux de dire au peuple que les lois ne sont pas justes, car il n'y obéit qu'à cause qu'il les croit justes. C'est pourquoi il faut lui dire en même temps qu'il y faut obéir parce qu'elles sont lois, comme il faut obéir aux supérieurs non pas parce qu'ils sont justes, mais parce qu'ils sont supérieurs. Par-là voilà toute sédition prévenue, si on peut faire entendre cela et que proprement c'est la définition de la justice. » (Fr. 100-66).

57. I. Adinolfi, Il cerchio spezzato, cit., pp. 108-9.

58. Ibid., p. 110.

59. D. Bosco, Sfumati pascaliani, cit., p. 23.

60. I. Adinolfi, Il cerchio spezzato, cit., p. 111.

61. Ibid., p. 115.

62. A. Del Noce, Riforma cattolica e filosofia moderna, Bologna, Il Mulino, 1965, p. 642.

63. C. Ciancio, "Pascal contro Cartesio: l'altro inizio del moderno", in AA. VV., L'incerto potere della ragione, cit., p. 18.

64. Ibid., p. 32.

65. G. Brescia, Pascal e l'ermeneutica, cit., p. 71.

66. Fr. $770-929$ et 927.

67. B. Razzotti, Pascal e i suoi problemi. Riflessioni filosofiche ed etico-politiche, cit., p. 172.

68. Ibid., p. 173.

69. Ibid., pp. 15-6.

70. Ibid., p. 190.

71. L. Pareyson, Kierkegaard e Pascal, Milano, Mursia, 1998. Ce volume contient trois essais de L. Pareyson : L'etica di Kierkegaard (1965) ; L'etica di Pascal (1966); et L'etica di Kierkegaard nella « Postilla (1971). Je désire rappeler l'attention du lecteur sur l'essai de L. Pareyson L'etica di Pascal, parce que, quoique étant le fruit d'une période précédente par rapport à celle que j'examine, il contient une des plus importantes réflexions sur l'éthique de Pascal.

72. Voir B. Razzotti, Pascal e i suoi problemi. Riflessioni filosofiche ed etico-politiche, cit., p. 18.

73. Ibid., p. 132.

74. Ibid., p. 135.

75. Ibid., pp. 132-3.

76. Cfr. Ibid., p. 133.

77. L. Pareyson, Kierkegaard e Pascal, cit., p. 192.

78. Ibid., p. 194.

79. Voir M. V. Romeo, Il numero e l'infinito, cit., pp. 368-9.

80. «Deux sortes de personnes connaissent, ceux qui ont le cœur humilié et qui aiment leur bassesse, quelque degré d'esprit qu'ils aient haut ou bas, ou ceux qui ont assez d'esprit pour voir la vérité quelques oppositions qu'ils y aient » (Fr. 13-394).

81. L. Pareyson, Kierkegaard e Pascal, cit., p. 216.

82. Ibid., p. 217.

83. Voir M. V. Romeo, Verità e bene, cit., p. 293-5.

84. L. Pareyson, Kierkegaard e Pascal, cit., p. 227.

85. Voir M. V. Romeo, Il numero e l'infinito, cit., pp. 382-84.

86. A. Peratoner, Blaise Pascal. Ragione, Rivelazione e Fondazione dell'Etica. Il percorso dell'Apologie, cit., p. 770.

87. M. V. Romeo, Il numero e l'infinito, cit., p. 371.

88. L. Pareyson, Kierkegaard e Pascal, cit., p. 271.

89. Ibid., p. 272.

90. «Malgré la vue de toutes nos misères qui nous touchent, qui nous tiennent à la gorge, nous avons un instinct que nous ne pouvons réprimer qui nous élève » (Fr. 527-633). 
91. Fr. 682-418. C'est moi qui souligne.

92. A. Peratoner, Blaise Pascal. Ragione, Rivelazione e Fondazione dell'Etica. Il percorso dell'Apologie, cit., p. 768.

93. Ibid., p. 788.

94. Fr. 78-45.

95. A. Peratoner, Blaise Pascal. Ragione, Rivelazione e Fondazione dell'Etica. Il percorso dell'Apologie, cit. p. 788.

96. Fr. 782-946. C'est moi qui souligne.

97. Fr. 648-793.

98. Fr. 773-931. C'est moi qui souligne.

99. G. Pezzino, « Prefazione » a AA. VV., L’incerto potere della ragione, cit., pp. 12-13.

\section{INDEX}

Mots-clés: Pascal, politique, éthique

Index géographique : Italie

Keywords : politics, ethics

\section{AUTEUR}

MARIA VITA ROMEO

Université de Catane

Chargée de recherche universitaire en philosophie morale 